\title{
Checklist of marine demersal fishes captured by the pair trawl fisheries in Southern (RJ-SC) Brazil
}

\author{
Matheus Marcos Rotundo ${ }^{1,2,3,4} \mathbb{B}^{-}$, Evandro Severino-Rodrigues ${ }^{2}$, Walter Barrella ${ }^{4,5}$, Miguel Petrere Jun- \\ ior $^{3}$ \& Milena Ramires ${ }^{4,5}$ \\ ${ }^{1}$ Universidade Santa Cecilia, Acervo Zoológico, R. Oswaldo Cruz, 266, CEP11045-907, Santos, SP, Brasil \\ ${ }^{2}$ Instituto de Pesca, Programa de Pós-graduação em Aquicultura e Pesca, Santos, SP, Brasil \\ ${ }^{3}$ Universidade Federal de São Carlos, Programa de Pós-Graduação em Planejamento e Uso de Recursos \\ Renováveis, Rodovia João Leme dos Santos, Km 110, CEP 18052-780, Sorocaba, SP, Brasil \\ ${ }^{4}$ Universidade Santa Cecília, Programa de Pós-Graduação de Auditoria Ambiental, R. Oswaldo Cruz, 266, \\ CEP11045-907, Santos, SP, Brasil \\ ${ }^{5}$ Universidade Santa Cecília, Programa de Pós-Graduação em Sustentabilidade de Ecossistemas Costeiros e \\ Marinhos, R. Oswaldo Cruz, 266, CEP11045-907, Santos, SP, Brasil
}

*Corresponding author: Matheus Marcos Rotundo: mmrotundo@unisanta.br ROTUNDO, M.M., SEVERINO-RODRIGUES, E., BARRELLA, W., PETRERE JUNIOR, M., RAMIRES, M. Checklist of marine demersal fishes captured by the pair trawl fisheries in Southern (RJ-SC) Brazil. Biota Neotropica. 19(1): e20170432. http://dx.doi.org/10.1590/1676-0611-BN-2017-0432

\begin{abstract}
Demersal fishery resources are abundant on continental shelves, on the tropical and subtropical coasts, making up a significant part of the marine environment. Marine demersal fishery resources are captured by various fishing methods, often unsustainably, which has led to the depletion of their stocks. In order to inventory the marine demersal ichthyofauna on the Southern Brazilian coast, as well as their conservation status and distribution, this study analyzed the composition and frequency of occurrence of fish captured by pair trawling in 117 fishery fleet landings based in the State of São Paulo between 2005 and 2012. The ichthyofauna consisted of 245 species (81 families, 32 orders and 2 classes). Among the species, 50 species were classified as constant, 38 accessory and 157 accidental. Still, $13.47 \%$ of the species were listed as endangered; $35.29 \%$ of the Chondrichthyes and $11.85 \%$ of the Actinopterygii (15.1\% of the total species) belong to the endemic fauna of the Biogeographic Province of Argentina. The richness was directly related to the oceanographic features of the study area, which determined the southern boundary of occurrence of several tropical species and the northern boundary of occurrence for temperate species. This is the region with the highest abundance of fishery resources in Brazil.
\end{abstract}

Keywords: ichthyofauna, inventory, fishery resources, conservation.

\section{Lista de peixes demersais marinhos capturados pela pesca de parelha no Sul (RJ-SC) do Brasil}

Resumo: Nas costas tropicais e subtropicais, sobre as plataformas continentais, os recursos pesqueiros demersais são abundantes e considerados uma parcela importante do ambiente marinho. Os recursos pesqueiros demersais marinhos são capturados por diversas modalidades de pesca, muitas vezes de forma insustentável, o que vem provocando o esgotamento de seus estoques. Com o objetivo de inventariar a ictiofauna demersal marinha da costa sudeste-sul do Brasil, assim como seu status de conservação e distribuição, foram analisadas a composição e frequência de peixes capturados em 117 cruzeiros de pesca entre 2005 e 2012 pela frota de arrasto de parelha sediada no Estado de São Paulo. A ictiofauna foi composta por 245 espécies ( 81 famílias, 32 ordens e 02 classes), sendo 50 consideradas como constantes, 38 acessórias e 157 acidentais; $13.47 \%$ apresentam algum grau de ameaça; $35.29 \%$ dos Chondrichthyes e $11.85 \%$ dos Actinopterygii (15.1\% do total) pertencem à fauna endêmica da Província Zoogeográfica da Argentina. A riqueza observada está diretamente vinculada às características oceanográficas da área de estudo, sendo estas responsáveis pelo limite meridional de ocorrência de várias espécies tropicais e o limite setentrional de espécies de regiões temperadas. Esta é a região com maior abundância de recursos pesqueiros do Brasil.

Palavras-chave: ictiofauna, inventário, recursos pesqueiros, conservação. 


\section{Introduction}

Demersal fishery resources are abundant on continental shelves, on the tropical and subtropical coasts, making up a significant part of the marine environment. Oceanographic conditions influence the quality and potential of such resources, as well as regionally limit the use of certain types of vessels and fishing gear (Yáñez-Arancibia \& SánchesGil 1988, Dias-Neto \& Ximenes 1998).

The capture of demersal species has been carried out by artisanal and industrial fishing for several decades and it is one of the most important activities for the fishing industry in Brazil (Castro et al. 2003). Smallscale fishing consists of beach seining, gillnets, long-lines, stern trawlers or double rig trawlers along estuarine and coastal and regions. Industrial fishing is carried out by medium and large-sized vessels using pair trawls and otter trawlnets, stern trawlers or double rig trawlers, gillnets and long-lines, as well as fishing with traps, coves and purse seines (Sudepe 1985, Valentini et al. 1991, Ibama 1993, 1995, Haimovici 1997).

The industrial pair trawling stands out among various modalities and categories of fishing aimed to demersal fish. Species belonging to the family Sciaenidae (Weakfishes), such as Micropogonias furnieri (Whitemouth croaker), Macrodon atricauda (Southern King Weakfish) and Cynoscion jamaicensis (Jamaica weakfish); in addition to species of the family Balistidae, such as Balistes capriscus (Grey triggerfish) and family Ariidae, such as Genidens barbus (White sea catfish) are the most targeted along with elasmobranchs (Valentini et al. 1991, Ibama 1993, 1995, Haimovici 1997, Castro et al. 2003, 2007, Castro \& Tutui 2007).

According to Caddy \& Sharp (1986), it is necessary to bear in mind that exploited species are linked to others that may be essential for the economic and biological productivity of the ecosystem. This is the only way to minimize the adverse effects of fishing and not to compromise the system's ability to sustain productive human activity. Therefore, the proper management of exploited resources and aquaculture requires knowledge about the dynamics of communities/populations, their ecological interactions, the integration with physicochemical and biological processes at spatial and temporal scales, as well as changes in fishery dynamics (Yáñes-Arancibia et al. 1985, Davis \& Anderson 1989, Aryuthaka \& Thumbthimsang 1992, Castelo et al. 2007, Castro \& Tutui 2007).

This study provides a checklist of marine demersal ichthyofauna of the southern coasts of Brazil captured by the fishery fleet pair trawling based in the state of São Paulo and comments upon the composition, frequency of occurrence, conservation status and geographical distribution of the recorded species.

\section{Material and Methods}

The pair trawling fleet operation area had its limits under the coordinates

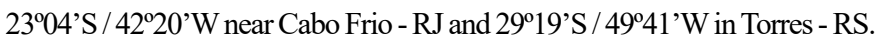
Prior to SMA-SP Resolution No. 69 of 2009, activities were concentrated on the south coast of São Paulo under the coordinates 2431'S / 46 $39^{\circ} \mathrm{W}$ near Peruíbe - SP and 28 $37^{\circ}$ S / 48 37'W near Cabo de Santa Marta - SC (Figure 1).

The fleet operated between a depth of 14 and 55 meters $(\mathrm{m} \pm \mathrm{SD}=$ $29.2 \pm 9.7 \mathrm{~m}$ ). For each year, the following means \pm standard deviation and amplitudes were observed: $30.4 \pm 8.6 \mathrm{~m}(22$ and $50 \mathrm{~m})$ in $2005 ; 27.9$ $\pm 7.5 \mathrm{~m}(20$ and $42 \mathrm{~m})$ in $2006 ; 33.2 \pm 13.9 \mathrm{~m}(14$ and $52 \mathrm{~m})$ in $2007 ; 25.2$ $\pm 11.9(15$ and $55 \mathrm{~m})$ in $2008 ; 28.5 \pm 10.9 \mathrm{~m}(14$ and $50 \mathrm{~m})$ in $2009 ; 30.1$ $\pm 9.7 \mathrm{~m}(26$ and $52 \mathrm{~m})$ in $2010 ; 30.4 \pm 6.3 \mathrm{~m} \mathrm{(26}$ and $50 \mathrm{~m})$ in 2011 ; and $30.9 \pm 6.6 \mathrm{~m}(26$ and $44 \mathrm{~m})$ in 2012.
The ichthyofauna from 117 fishery fleet landings of the industrial pair trawling fleet based in the State of São Paulo were sampled from March 2005 to May 2012. The sampling order was randomly. Only landings at the ports located in the municipalities of Santos and GuarujáSP, Brazil were recorded.

The following sampling routine was performed for qualitative purposes: (a) the whole screening operation of the marketable product was followed up by collecting all rejected material among commercial products and also part of this products; (b) samples of the rejected and discarded products that fishermen sampled during the whole fishing operation were collected for further evaluation in the laboratory; c) co-ordinates of the most distant bids, as well as of the main fishing grounds and their respective depths, were annotated with the masters. Samples of rejected and discarded fauna were also obtained through 15 shipments to the studied fleet.

The collected specimens were referred to the laboratory in iceboxes. Samples were identified, counted and had their total length measured (in $\mathrm{mm})$. Subsequently, the specimens were fixed in $10 \%$ formaldehyde and preserved in $70 \%$ ethyl alcohol. Voucher specimens of the some species collected during the study were deposited in the regional collection of fish from the Atlantic Forest coast of the "Acervo Zoológico da Universidade Santa Cecília" (AZUSC), in the city of Santos-SP, Brazil.

Fish species in the samples were identified according to Figueiredo (1977), Figueiredo \& Menezes (1978, 1980, 2000), Fischer (1978), Menezes \& Figueiredo (1980, 1985), Compagno (1984, 1988), Cervigón et al. (1992), Carvalho-Filho (1999), Moura et al. (2001), Carpenter (2002), Fischer et al. (2004), Marceniuk (2005), Leis (2006), Moura \& Lindeman (2007), Sampaio \& Nottingham (2008), Carvalho-Filho et al. (2010), Gomes et al. (2010), McBride et al. (2010), Rosa \& Gadig (2010), Tavera et al. (2011, 2012), Ruocco et al. (2012), Frable et al. (2013), Menezes et al. (2015), Knudsen \& Clements (2016), Last et al. (2016a, b, c); Marceniuk et al. (2016). The systematics and nomenclature followed Nelson et al. (2016) and Eschmeyer et al. (2017), respectively.

Species were classified from their frequencies of occurrence and according to the Dajoz's (1983) scale as "constant" when present in more than $50 \%$ of the samples; "accessory" when frequencies ranged from 25 to $50 \%$ and "accidental" when frequencies were below $25 \%$.

The conservation status of each species were based on the International Red List of threatened species (IUCN - International Union for Conservation of Nature 2017) and the Brazilian Federal list of threatened species, Ordinance number 445 of the MMA - Brazilian Environment Ministry, December 17th 2014 (MMA 2014).

Species were classified in the biogeographical categories proposed by Floeter et al. (2008) and Luiz Jr. et al. (2008).

\section{Results}

Of the 117 fishery fleet landings, the majority occurred in 2011 $(20.52 \%)$ and the minority in 2010 (5.98\%) (Table 1).

The richness consisted of 245 species (Table 2) distributed over 2 classes, 32 orders, 81 families and 170 genera. Out of the total species analyzed, $86.12 \%$ of the species belong to Class Actinopterygii and $13.88 \%$ to Class Chondrichthyes (Table 3). Of the 81 families comprising the captured ichthyofauna, six families had the highest species richness $(33.5 \%$ of the total), namely Carangidae (6), Sciaenidae (19), Paralichthyidae (11), Haemulidae and Serranidae (10) and Engraulidae (8). 


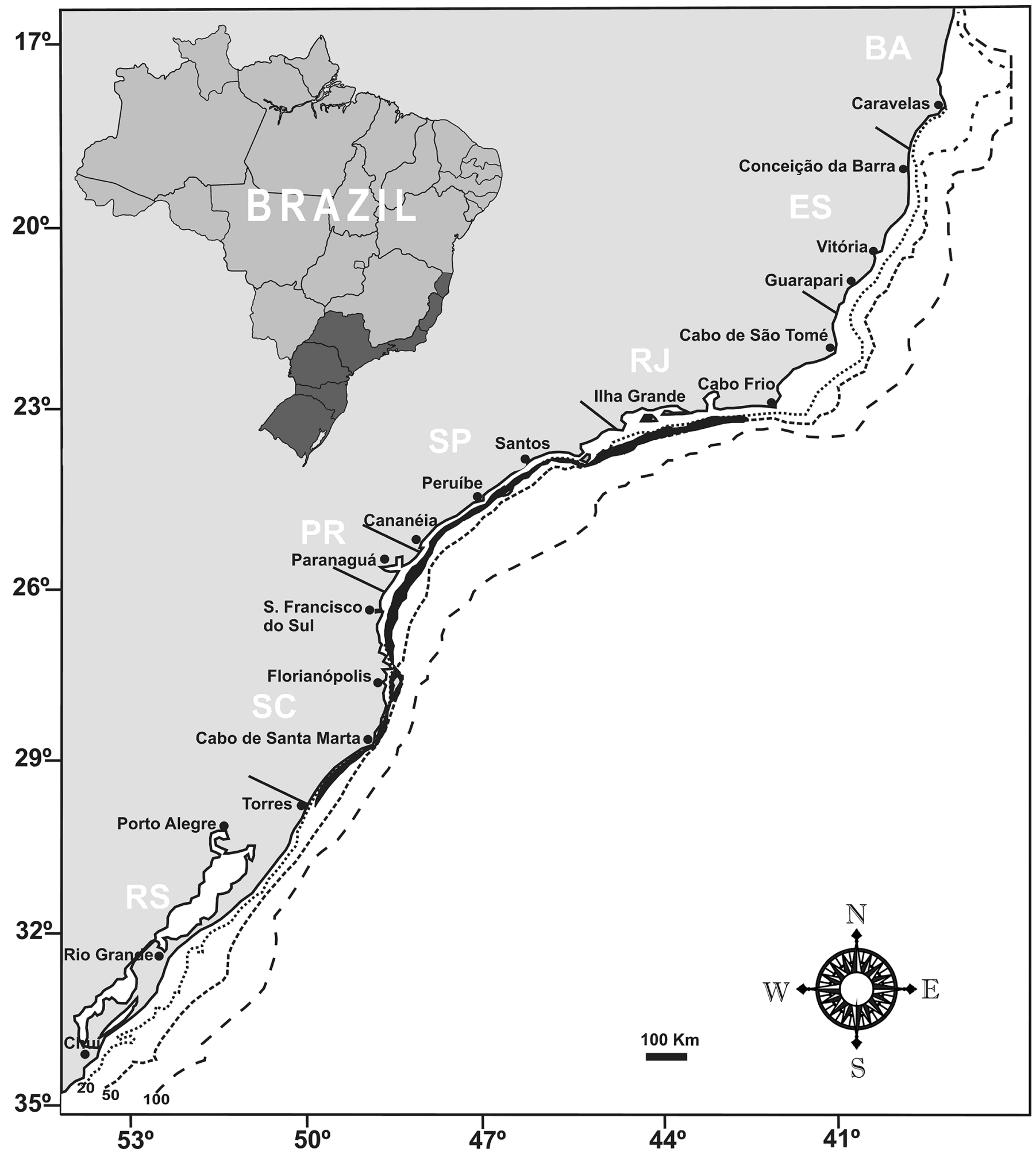

Figure 1. Areas of legal practice of pair trawling fishing in the State of São Paulo (Espírito Santo to Rio Grande do Sul). The study area is highlighted in black.

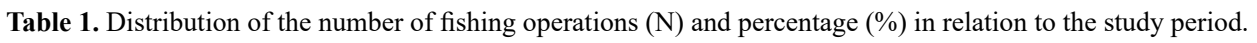

\begin{tabular}{ccccccccccc}
\hline & $\mathbf{2 0 0 5}$ & $\mathbf{2 0 0 6}$ & $\mathbf{2 0 0 7}$ & $\mathbf{2 0 0 8}$ & $\mathbf{2 0 0 9}$ & $\mathbf{2 0 1 0}$ & $\mathbf{2 0 1 1}$ & $\mathbf{2 0 1 2}$ & Total \\
\hline $\mathrm{N}$ & 10 & 17 & 13 & 18 & 20 & 7 & 24 & 8 & 117 \\
$\%$ & 8.5 & 14.5 & 11.1 & 15.3 & 17.0 & 5.9 & 20.5 & 6.8 & 100 \\
\hline
\end{tabular}


Table 2. Ichthyofauna caught by the pair trawling fleet of the state of São Paulo, Dajoz scale $(\mathrm{DS})$ : Co $=$ constant, Ac $=$ accessory, Ad = accidental; geographic distribution (GD): CT = circumtropical, TA = Trans-Atlantic (both sides of the Atlantic), WA = Western Atlantic (Northern and Southwestern Atlantic), SWA = Southern West Atlantic (from northern Brazil to Argentina), SSWA= Southern South West Atlantic (species with affinity to temperate areas occurring from Argentina

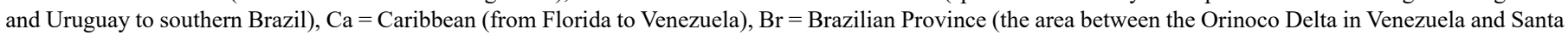
Catarina in Brazil) and EP = Eastern Pacific; conservation status in the IUCN Red List (IUCN 2017), in the Brazilian list (MMA 2014): X = Not Evaluated; LC = Least Concern; DD = Data Deficient; VU = Vulnerable; NT = Near-Threatened; END = Endangered, CR = Critically Endangered; vouchers.

\begin{tabular}{|c|c|c|c|c|c|}
\hline Order/ Family/ Species & DS & GD & IUCN & MMA & Voucher \\
\hline \multicolumn{6}{|l|}{ CARCHARHINIFORMES } \\
\hline \multicolumn{6}{|l|}{ Triakidae } \\
\hline Mustelus schmitti Springer, 1939 & Ad & SSWA & END & $\mathrm{CR}$ & AZUSC3405 \\
\hline \multicolumn{6}{|l|}{ Carcharhinidae } \\
\hline Carcharhinus brachyurus (Günther, 1870) & $\mathrm{Ad}$ & $\mathrm{CT}$ & NT & & AZUSC3791 \\
\hline Carcharhinus falciformis (Bibron, 1839) & $\mathrm{Ad}$ & $\mathrm{CT}$ & $\mathrm{VU}$ & & AZUSC3354 \\
\hline Carcharhinus limbatus (Valenciennes, 1839) & Ad & $\mathrm{CT}$ & NT & & AZUSC 3350 \\
\hline Galeocerdo cuvier (Péron \& Lesueur, 1822) & Ad & $\mathrm{CT}$ & NT & & AZUSC3383 \\
\hline Rhizoprionodon lalandii (Valenciennes, 1839) & Co & WA & DD & & AZUSC3293 \\
\hline Rhizoprionodon porosus (Poey, 1861) & $\mathrm{Ad}$ & $\mathrm{Ca}+\mathrm{SWA}$ & LC & & AZUSC4811 \\
\hline \multicolumn{6}{|l|}{ Sphyrnidae } \\
\hline Sphyrna lewini (Griffith \& Smith, 1834) & $\mathrm{Ad}$ & $\mathrm{CT}$ & END & $\mathrm{CR}$ & AZUSC0893 \\
\hline Sphyrna zygaena (Linnaeus, 1758) & $\mathrm{Ad}$ & $\mathrm{CT}$ & VU & $\mathrm{CR}$ & AZUSC3780 \\
\hline
\end{tabular}

SQUATINIFORMES

\section{Squatinidae}

Squatina guggenheim Marini, 1936

Ac

SSWA

END

CR

AZUSC3203

TORPEDINIFORMES

\section{Torpedinidae}

Tetronarce puelcha (Lahille, 1926)

Ad

Narcinidae

Narcine brasiliensis (Olfers, 1831)

RAJIFORMES

\section{Rajidae}

Atlantoraja castelnaui (Miranda Ribeiro, 1907)

Atlantoraja cyclophora (Regan, 1903)

Atlantoraja platana (Günther, 1880)

Psammobatis extenta (Garman, 1913)

Psammobatis lentiginosa McEachran, 1983

Rioraja agassizii (Müller \& Henle, 1841)

Sympterygia bonapartii Müller \& Henle, 1841

PRISTIFORMES

\section{Rhinobatidae}

Pseudobatos horkelii (Müller \& Henle, 1841)

Pseudobatos percellens (Walbaum, 1792)

Zapteryx brevirostris (Müller \& Henle, 1841)

MYLIOBATIFORMES

\section{Dasyatidae}

Hypanus americanus (Hildebrand \& Schroeder, 1928)

Dasyatis hypostigma Santos \& Carvalho, 2004

Hypanus guttatus (Bloch \& Schneider,1801)

Pteroplatytrygon violacea (Bonaparte, 1832)
Ac

Ad

Ad

Ad

Ad

Co

Ad

Ac

Co

Co

Ac

Co

Ad

Ad
SSWA

WA

SSWA

SSWA

SSWA

SSWA

SSWA

SSWA

SSWA

SSWA

TA

WA

WA

SSWA

$\mathrm{Ca}+\mathrm{Br}$

CT
END

VU

VU

LC

DD

VU

DD

END

END

CR

AZUSC3454

AZUSC3456

NT

VU
AZUSC3147

AZUSC3346

AZUSC3349

AZUSC3446

AZUSC3442

AZUSC3432

AZUSC3782 


\section{Gymnuridae}

Gymnura altavela (Linnaeus, 1758)

Co

Myliobatidae

Aetobatus narinari (Euphrasen, 1790)

Mobula hypostoma (Bancroft, 1831)

Mobula thurstoni (Lloyd, 1908)

Myliobatis freminvillei Lesueur, 1824

Myliobatis goodei Garman, 1885

Rhinoptera bonasus (Mitchill, 1815)

Rhinoptera brasiliensis Müller, 1836

ELOPIFORMES

\section{Elopidae}

Elops smithi McBride, Rocha, Ruiz-Carus \& Bowen, 2010

Ad

ALBULIFORMES

\section{Albulidae}

Albula vulpes (Linnaeus, 1758)

Ac

ANGUILLIFORMES

\section{Muraenidae}

Gymnothorax conspersus Poey, 1867

Gymnothorax funebris Ranzani, 1839

Gymnothorax moringa (Cuvier, 1829)

Gymnothorax ocellatus Agassiz, 1831

Muraena retifera Goode \& Bean, 1882

\section{Ophichthidae}

Echiophis intertinctus (Richardson, 1848)

Ophichthus ophis (Linnaeus, 1758)

Ophichthus gomesii (Castelnau, 1855)

Muraenosocidae

Cynoponticus savanna (Bancroft, 1831)

Congridae

Conger orbignianus Valenciennes, 1837

CLUPEIFORMES

Pristigasteridae

Chirocentrodon bleekerianus (Poey, 1867)

Pellona harroweri (Fowler, 1917)

Engraulidae

Anchoa filifera (Fowler, 1915)

Anchoa marinii Hildebrand, 1943

Anchoa spinifer (Valenciennes, 1848)

Anchoa tricolor (Spix \& Agassiz, 1829)

Anchovia clupeoides (Swainson, 1839)

Anchoviella lepidentostole (Fowler, 1911)

Engraulis anchoita Hubbs \& Marini, 1935

Lycengraulis grossidens (Spix \& Agassiz, 1829)

Clupeidae

Brevoortia pectinata (Jenyns, 1842)

Harengula clupeola (Cuvier, 1829)

Ad

Ad

Ad

Ad

Ad

Ad

Ad

Ad

Ad

Ad

Ac

Ad

Ad

Ad

Ac

Ad

Ad

Ac

Ad

Ad

Ad

Ad

Ad

Ad

Ad

Ad

Ad

Co
TA

WA

DD

SSWA

$\mathrm{Ca}+\mathrm{Br}$

$\mathrm{Ca}+\mathrm{Br}$

LC

LC

$\mathrm{Ca}+\mathrm{Br}$

SSWA

$\mathrm{Ca}+\mathrm{Br}+\mathrm{EP}$

SWA

$\mathrm{Ca}+\mathrm{Br}$

$\mathrm{Br}$

SSWA

$\mathrm{Br}+\mathrm{SSWA}$

LC

LC

LC

LC

LC

LC

$\mathrm{X}$

LC

SSWA

LC

LC
AZUSC1360

AZUSC1742

AZUSC3387

AZUSC3291

AZUSC3400

AZUSC3403

AZUSC3412

AZUSC3418

AZUSC3783

AZUSC0877

AZUSC3719

AZUSC2108

AZUSC2710

AZUSC2010

AZUSC2299

AZUSC2109

AZUSC2256

AZUSC3673

AZUSC3761

AZUSC1120

AZUSC1488

AZUSC1477

AZUSC1490

AZUSC4620

AZUSC4621

AZUSC4625

AZUSC1476

AZUSC1487

AZUSC2160

AZUSC4663

AZUSC3897

AZUSC1328 
Rotundo, M.M. et al.

Opisthonema oglinum (Lesueur, 1818)

Sardinella brasiliensis (Steindachner, 1879)

\section{SILURIFORMES}

Ariidae

Aspistor luniscutis (Valenciennes, 1840)

Bagre bagre (Linnaeus, 1766)

Bagre marinus (Mitchill, 1815)

Cathorops spixii (Agassiz, 1829)

Genidens barbus (Lacepède, 1803)

Genidens genidens (Cuvier, 1829)

AULOPIFORMES

Synodontidae

Synodus bondi Fowler, 1939

Trachinocephalus myops (Forster, 1801)

GADIFORMES

Merlucciidae

Merluccius hubbsi Marini, 1933

Gadidae

Urophycis brasiliensis (Kaup, 1858)

HOLOCENTRIFORMES

\section{Holocentridae}

Holocentrus adscensionis (Osbeck, 1765)

\section{OPHIDIIFORMES}

\section{Ophidiidae}

Genypterus brasiliensis Regan, 1903

Ophidion holbrookii Putnam, 1874

Raneya brasiliensis (Kaup, 1856)

BATRACHOIDIFORMES

Batrachoididae

Opsanus beta (Goode \& Bean, 1880)

Porichthys porosissimus (Cuvier, 1829)

Thalassophryne montevidensis (Berg, 1893)

MUGILIFORMES

\section{Mugilidae}

Mugil curema Valenciennes, 1836

Mugil liza Valenciennes, 1836

\section{Polynemidae}

Polydactylus oligodon (Günther, 1860)

Polydactylus virginicus (Linnaeus, 1758)

\section{BELONIFORMES}

\section{Exocoetidae}

Cheilopogon melanurus (Valenciennes, 1847)

\section{Hemiramphidae}

Hemiramphus balao Lesueur, 1821

Hemiramphus brasiliensis (Linnaeus, 1758)

Hyporhamphus unifasciatus (Ranzani, 1841)
Ad

Ad

WA

SSWA

LC

$\mathrm{X}$

AZUSC1327

AZUSC2164

$\begin{array}{lll}\text { Co } & \mathrm{Br} & \mathrm{X}\end{array}$

$\mathrm{Ac} \quad \mathrm{Ca}+\mathrm{Br} \quad \mathrm{LC}$

Ad

WA

$\mathrm{Ca}+\mathrm{Br}$

Ad

$\mathrm{Br}+\mathrm{SSWA}$

$\mathrm{Br}+\mathrm{SSWA}$

WA

CT

LC

AZUSC2090

Ac

Ad

SSWA

X

AZUSC1288

Ad

SSWA

X

AZUSC2122

Ad

TA

LC

AZUSC1699
Ad

Ac

Ac

Ad

Co

Ad

Ad

Ad

Ad

Ac

Ad

Ad

Ad

Ad
SSWA

WA

SSWA

$\mathrm{Ca}$

SSWA

SWA

TA+EP

WA

WA

WA

TA

TA

TA

$\mathrm{WA}+\mathrm{EP}$
X

LC

X

CR

AZUSC0905

AZUSC1352

AZUSC1811

AZUSC3144

AZUSC1146

AZUSC3088

AZUSC1814

AZUSC2698

AZUSC2089

AZUSC2011
AZUSC4008

AZUSC4604

AZUSC4618 
Marine demersal fishes in Southern Brazil.

\section{Belonidae}

Ablennes hians (Valenciennes, 1846)

Strongylura marina (Walbaum, 1792)

Strongylura timucu (Walbaum, 1792)

Tylosurus acus (Lacepède, 1803)

CARANGIFORMES

\section{Coryphaenidae}

Coryphaena hippurus Linnaeus, 1758

Rachycentridae

Rachycentron canadum (Linnaeus, 1766)

Echeneidae

Echeneis naucrates Linnaeus, 1758

Carangidae

Alectis ciliaris (Bloch, 1787)

Caranx crysos (Mitchill, 1815)

Caranx hippos (Linnaeus, 1766)

Caranx latus Agassiz, 1831

Chloroscombrus chrysurus (Linnaeus, 1766)

Decapterus punctatus (Cuvier, 1829)

Hemicaranx amblyrhynchus (Cuvier, 1833)

Oligoplites palometa (Cuvier, 1832)

Oligoplites saliens (Bloch, 1793)

Oligoplites saurus (Bloch \& Schneider, 1801)

Parona signata (Jenyns, 1841)

Pseudocaranx dentex (Bloch \& Schneider, 1801)

Selar crumenophthalmus (Bloch, 1793)

Selene setapinnis (Mitchill, 1815)

Selene vomer (Linnaeus, 1758)

Seriola dumerili (Risso, 1810)

Seriola fasciata (Bloch, 1793)

Seriola lalandi Valenciennes, 1833

Trachinotus carolinus (Linnaeus, 1766)

Trachinotus falcatus (Linnaeus, 1758)

Trachinotus goodei Jordan \& Evermann, 1896

Trachinotus marginatus Cuvier, 1832

Trachurus lathami Nichols, 1920

Uraspis secunda (Poey, 1860)

ISTIOPHORIFORMES

\section{Sphyraenidae}

Sphyraena guachancho Cuvier, 1829

Sphyraena tome Fowler, 1903

Xiphiidae

Xiphias gladius Linnaeus, 1758

PLEURONECTIFORMES

Paralichthyidae

Citharichthys arenaceus Evermann \& Marsh, 1900

Citharichthys macrops Dresel, 1885
Ad

CT

WA

WA

TA

Ad

Ad

Ad

Ad

Ad

Ac

Ac

Ad

Co

Ad

Ac

Ad

Co

Ad

Ad

Ac

Ad

Co

$\mathrm{Co}$

Ad

Ad

Ad

Co

Ad

Ac

Ad

Ad

Ad

Co

Ac

Ad

Ac

$\mathrm{Ad}$
LC

LC

LC

LC

LC

AZUSC4601

AZUSC2244

AZUSC3232

AZUSC1389

AZUSC1319

AZUSC1658

AZUSC1322

AZUSC1344

AZUSC1792

AZUSC1928

AZUSC3037

AZUSC1317

AZUSC3771

AZUSC3604

AZUSC1329

AZUSC2447

AZUSC1323

AZUSC1388

AZUSC3668

AZUSC3677

AZUSC2634

AZUSC1336

AZUSC3149

AZUSC2016

AZUSC2678

AZUSC1122

AZUSC1963

AZUSC1341

AZUSC1602

AZUSC4598 
Cyclopsetta chittendeni Bean, 1895

Etropus crossotus Jordan \& Gilbert, 1882

Etropus longimanus Norman, 1933

Paralichthys brasiliensis (Ranzani, 1842)

Paralichthys orbignyanus (Valenciennes, 1839)

Paralichthys patagonicus Jordan, 1889

Syacium papillosum (Linnaeus, 1758)

Syacium micrurum Ranzani, 1842

Xystreurys rasile (Jordan, 1891)

\section{Bothidae}

Bothus ocellatus (Agassiz, 1831)

Bothus robinsi Topp \& Hoff, 1972

Achiridae

Achirus declivis Chabanaud, 1940

Gymnachirus nudus Kaup, 1858

Trinectes paulistanus (Miranda Ribeiro, 1915)

\section{SYNGNATHIFORMES}

\section{Fistulariidae}

Fistularia petimba Lacepède, 1803

Fistularia tabacaria Linnaeus, 1758

\section{Dactylopteridae}

Dactylopterus volitans (Linnaeus, 1758)

SCOMBRIFORMES

\section{Gempylidae}

Thyrsitops lepidopoides (Cuvier, 1832)

\section{Trichiuridae}

Evoxymetopon taeniatus Gill, 1863

Trichiurus lepturus Linnaeus, 1758

Scombridae

Scomber japonicus Houttuyn, 1782

Scomberomorus brasiliensis Collette, Russo \& ZavalaCamin, 1978

Scomberomorus cavalla (Cuvier, 1829)

Thunnus alalunga (Bonnaterre, 1788)

\section{Centrolophidae}

Seriolella porosa Guichenot, 1848

\section{Stromateidae}

Peprilus xanthurus (Quoy \& Gaimard, 1825)

TRACHINIFORMES

\section{Pinguipedidae}

Pseudopercis semifasciata (Cuvier, 1829)

\section{Percophidae}

Percophis brasiliensis Quoy \& Gaimard, 1825

\section{Uranoscopidae}

Astroscopus sexspinosus (Steindachner, 1876)

Astroscopus y-graecum (Cuvier, 1829)

LABRIFORMES

$\begin{array}{lccc}\text { Ad } & \text { Ca+Br } & \text { LC } & \text { AZUSC1651 } \\ \text { Ad } & \text { WA+EP } & \text { LC } & \text { AZUSC2159 } \\ \text { Ad } & \text { SSWA } & \text { X } & \text { AZUSC4622 } \\ \text { Ac } & \text { SWA } & \text { X } & \text { AZUSC1521 } \\ \text { Ad } & \text { SSWA } & \text { X } & \text { AZUSC1926 } \\ \text { Ac } & \text { SSWA+EP } & \text { X } & \text { AZUSC1392 } \\ \text { Ad } & \text { WA } & \text { LC } & \text { AZUSC1549 } \\ \text { Ad } & \text { WA } & \text { LC } & \text { AZUSC3036 } \\ \text { Ad } & \text { SSWA } & \text { X } & \text { AZUSC4600 } \\ & & & \\ \text { Ad } & \text { WA } & \text { LC } & \text { AZUSC3086 } \\ \text { Ad } & \text { WA } & \text { LC } & \text { AZUSC2088 } \\ & & \text { LC } & \text { AZUSC2245 } \\ \text { Ad } & \text { WA } & \text { LC } & \text { AZUSC1949 } \\ \text { Ac } & \text { WA } & \text { LC } & \text { AZUSC4664 } \\ \text { Ad } & \text { Ca+Br } & & \end{array}$

Ad

TA

LC

AZUSC1330

Co

TA

$\mathrm{LC}$

AZUSC2018

Co

TA

LC

AZUSC1147

Ad

SSWA

$\mathrm{X}$

AZUSC1148

Ad

$\mathrm{Ca}+\mathrm{SWA}+\mathrm{EP}$

LC

AZUSC2939

Co

CT

LC

AZUSC1391

Ad

CT

LC

AZUSC2286

Co

$\mathrm{Ca}+\mathrm{Br}$

LC

AZUSC1369

Ad

WA

LC

AZUSC4599

Ad

CT

NT

AZUSC1933

$\mathrm{SWA}+\mathrm{EP}$

X

AZUSC2636

Co

SWA

X

AZUSC1333

Ad

SSWA

X

AZUSC2119

Ad

SSWA

X

AZUSC1145

Ad

SSWA

X

AZUSC2113

Ad

LC 


\section{Labridae}

Xyrichtys novacula (Linnaeus, 1758)

Scaridae

Nicholsina usta (Valenciennes, 1840)

Sparisoma axillare (Steindachner, 1878)

Sparisoma frondosum (Agassiz, 1831)

PERCIFORMES

\section{Centropomidae}

Centropomus parallelus Poey, 1860

Centropomus undecimalis (Bloch, 1792)

\section{Gerreidae}

Diapterus auratus Ranzani, 1842

Diapterus rhombeus (Cuvier, 1829)

Eucinostomus argenteus Baird \& Girard, 1855

Eucinostomus gula (Quoy \& Gaimard, 1824)

Eucinostomus melanopterus (Bleeker, 1863)

Eugerres brasilianus (Cuvier, 1830)

Polyprionidae

Polyprion americanus (Bloch \& Schneider, 1801)

Mullidae

Pseudupeneus maculatus (Bloch, 1793)

Upeneus parvus Poey, 1852

Kyphosidae

Kyphosus vaigiensis (Quoy \& Gaimard, 1825)

Kyphosus sectatrix (Linnaeus, 1758)

Serranidae

Diplectrum formosum (Linnaeus, 1766)

Diplectrum radiale (Quoy \& Gaimard, 1824)

Dules auriga Cuvier, 1829

Epinephelus itajara (Lichtenstein, 1822)

Epinephelus marginatus (Lowe, 1834)

Epinephelus morio (Valenciennes, 1828)

Hyporthodus niveatus (Valenciennes, 1828)

Mycteroperca acutirostris (Valenciennes, 1828)

Rypticus randalli Courtenay, 1967

Serranus atrobranchus (Cuvier, 1829)

Pomatomidae

Pomatomus saltatrix (Linnaeus, 1766)

Priacanthidae

Priacanthus arenatus Cuvier, 1829

Pomacanthidae

Pomacanthus paru (Bloch, 1787)

Malacanthidae

Caulolatilus chrysops (Valenciennes, 1833)

Haemulidae

Anisotremus surinamensis (Bloch, 1791)

Anisotremus virginicus (Linnaeus, 1758)
Ad

Ad

$\mathrm{Ad}$

Ad

Ad

Ac

Ad

Co

Ac

Ad

Ad

Ad

Ad

Ad

Ac

Ad

Ad

$\mathrm{Co}$

Ac

Ad

Ad

Ad

Ad

Ac

Ad

Ad

Ad

Co

Co

Ad

Ad

Ad

Ac
WA

WA

$\mathrm{Br}$

$\mathrm{Ca}+\mathrm{Br}$

WA

WA

LC

LC

LC

LC

LC

$\mathrm{LC}$

LC

LC

DD

LC

LC

LC

LC

LC

LC

WA

SSWA

WA

$\mathrm{WA}+\mathrm{EP}$

WA

WA

$\mathrm{Ca}+\mathrm{Br}$

$\mathrm{Ca}+\mathrm{Br}$

WA

CT

TA

WA

WA

WA

WA
C

AZUSC1157

AZUSC3644

AZUSC1828

AZUSC1799

AZUSC1372

AZUSC1363

AZUSC3898

AZUSC1325

AZUSC1326

AZUSC1359

AZUSC1625

AZUSC1875

CR

AZUSC3656

AZUSC1661

AZUSC1351

AZUSC3867

AZUSC0372

AZUSC1331

AZUSC1355

AZUSC1140

CR AZUSC1043

VU AZUSC2377

VU

AZUSC3646

VU

AZUSC1600

AZUSC1646

AZUSC3721

AZUSC3676

AZUSC1630

AZUSC1339

AZUSC1342

AZUSC1605

AZUSC1688

AZUSC1343 
Boridia grossidens Cuvier, 1830

Conodon nobilis (Linnaeus, 1758)

Genyatremus cavifrons (Cuvier, 1830)

Haemulon aurolineatum Cuvier, 1830

Haemulon steindachneri (Jordan \& Gilbert, 1882)

Orthopristis ruber (Cuvier, 1830)

Haemulopsis corvinaeformis (Steindachner, 1868)

Pomadasys crocro (Cuvier, 1830)

\section{Lutjanidae}

Lutjanus analis (Cuvier, 1828)

Lutjanus griseus (Linnaeus, 1758)

Lutjanus jocu (Bloch \& Schneider, 1801)

Lutjanus synagris (Linnaeus, 1758)

Rhomboplites aurorubens (Cuvier, 1829)

SCORPAENIFORMES

\section{Scorpaenidae}

Scorpaena brasiliensis Cuvier, 1829

Scorpaena isthmensis Meek \& Hildebrand, 1928

Scorpaena plumieri Bloch, 1789

Triglidae

Prionotus nudigula Ginsburg, 1950

Prionotus punctatus (Bloch, 1793)

MORONIFORMES

\section{Ephippidae}

Chaetodipterus faber (Broussonet, 1782)

\section{ACANTHURIFORMES}

\section{Sciaenidae}

Ctenosciaena gracilicirrhus (Metzelaar, 1919)

Cynoscion acoupa (Lacepède, 1801)

Cynoscion guatucupa (Cuvier, 1830)

Cynoscion jamaicensis (Vaillant \& Bocourt, 1883)

Cynoscion leiarchus (Cuvier, 1830)

Cynoscion virescens (Cuvier, 1830)

Isopisthus parvipinnis (Cuvier, 1830)

Larimus breviceps Cuvier, 1830

Macrodon atricauda (Günther, 1880)

Menticirrhus americanus (Linnaeus, 1758)

Menticirrhus littoralis (Holbrook, 1847)

Micropogonias furnieri (Desmarest, 1823)

Nebris microps Cuvier, 1830

Paralonchurus brasiliensis (Steindachner, 1875)

Pogonias cromis (Linnaeus, 1766)

Stellifer brasiliensis (Schultz, 1945)

Stellifer rastrifer (Jordan, 1889)

Umbrina canosai Berg, 1895

Umbrina coroides Cuvier, 1830

$\begin{array}{ccc}\text { Ad } & \text { SSWA } & \text { X } \\ \text { Co } & \text { WA } & \text { LC } \\ \text { Ad } & \text { Ca+Br } & \text { DD } \\ \text { Ac } & \text { WA } & \text { LC } \\ \text { Ac } & \text { Ca+SWA } & \text { LC } \\ \text { Co } & \text { Ca+SWA } & \text { LC } \\ \text { Co } & \text { Ca+SWA } & \text { LC } \\ \text { Ad } & \text { WA } & \text { DD } \\ & & \\ \text { Ad } & \text { WA } & \text { NT } \\ \text { Ad } & \text { WA } & \text { LC } \\ \text { Ad } & \text { WA } & \text { DD } \\ \text { Ad } & \text { WA } & \text { NT } \\ \text { Ad } & \text { WA } & \text { VU }\end{array}$

AZUSC1912

AZUSC1320

AZUSC1968

AZUSC1398

AZUSC1913

AZUSC1138

AZUSC1345

AZUSC3099

AZUSC2789

AZUSC 3100

AZUSC4542

AZUSC4321

AZUSC1423

Ac

Ad

Ac

Ad

Co

Co

Co

Ad

Ac

Co

Co

Co

$\mathrm{Co}$

Co

Co

Co

Ad

Co

Ac

Co

Ad

Ad

Ad

Ad

Ad
WA

WA

WA

SSWA

$\mathrm{Ca}+\mathrm{SWA}$

WA

LC

AZUSC1334

AZUSC1142

AZUSC3202

AZUSC1143

AZUSC1386

AZUSC1966

AZUSC1358

AZUSC1390

AZUSC1318

AZUSC1498

AZUSC1430

AZUSC4730

AZUSC1144

AZUSC1467

AZUSC1425

AZUSC3206

AZUSC1486

AZUSC 1520

AZUSC1123

AZUSC1365 
Marine demersal fishes in Southern Brazil.

\section{Acanthuridae}

Acanthurus chirurgus (Bloch, 1787)

SPARIFORMES

\section{Lobotidae}

Lobotes surinamensis (Bloch, 1790)

Sparidae

Archosargus aries (Valenciennes, 1830)

Archosargus rhomboidalis (Linnaeus, 1758)

Calamus penna (Valenciennes, 1830)

Calamus pennatula Guichenot, 1868

Diplodus argenteus (Valenciennes, 1830)

Pagrus pagrus (Linnaeus, 1758)

\section{LOPHIIFORMES}

\section{Lophiidae}

Lophius gastrophysus Miranda Ribeiro, 1915

\section{Antennariidae}

Antennarius striatus (Shaw, 1794)

Ogcocephalidae

Ogcocephalus vespertilio (Linnaeus, 1758)

\section{TETRAODONTIFORMES}

Ostraciidae

Acanthostracion quadricornis (Linnaeus, 1758)

Balistidae

Balistes capriscus Gmelin, 1789

Monacanthidae

Aluterus monoceros (Linnaeus, 1758)

Aluterus heudelotii Hollard, 1855

Stephanolepis hispidus (Linnaeus, 1766)

Molidae

Mola mola (Linnaeus, 1758)

Tetraodontidae

Lagocephalus laevigatus (Linnaeus, 1766)

Lagocephalus lagocephalus (Linnaeus, 1758)

Sphoeroides greeleyi Gilbert, 1900

Sphoeroides spengleri (Bloch, 1785)

Sphoeroides testudineus (Linnaeus, 1758)

Diodontidae

Chilomycterus spinosus (Linnaeus, 1758)
Ad

Ad

Ad

Ac

$\mathrm{Ad}$

Ad

Co

Co

Ad

Ad

Ac

Ad

Co

Ac

Ad

Co

Ad

Co

Ad

Ad

Ad

Ad

Co
TA

CT

WA

WA

WA

WA

SWA

TA

WA

WA

$\mathrm{Ca}+\mathrm{SWA}$

WA

LC

AZUSC2117

AZUSC1347

AZUSC1362

AZUSC4517

AZUSC1332

AZUSC3950

CT VU

TA

CT

$\mathrm{Ca}+\mathrm{Br}$

TA

WA

SWA
LC

LC

LC

LC

LC

LC
AZUSC1373

AZUSC3234

AZUSC2522

AZUSC3330

AZUSC2163

AZUSC1139

Table 3. Total number of ichthyofaunal taxa captured by the pair trawling fleet based in the State of São Paulo.

\begin{tabular}{ccc}
\hline Class & Chondrichthyes & Actinopterygii \\
\hline Order & 6 & 26 \\
Family & 11 & 70 \\
Genus & 22 & 148 \\
Species & 34 & 211 \\
\hline
\end{tabular}


From the frequency of occurrence (FO) and the classification proposed by Dajoz (1983) the ichthyofauna consisted of 50 species (20.41\%) classified as constants; 38 (15.5\%) accessory species and $157(64.08 \%)$ accidental species. Seventeen species $(6.34 \%)$ had $100 \%$ frequency throughout the sample period: Dactylopterus volitans (Flying gurnard), Prionotus punctatus (Bluewing searobin), Chloroscombrus chrysurus (Atlantic bumper), Oligoplites saliens (Castin leatherjacket), Selene setapinnis (Atlantic moonfish), S. vomer (Lookdown), Conodon nobilis (Barred grunt), Orthopristis ruber (Corocoro grunt), Diplodus argenteus (South American silver porgy), Cynoscion jamaicensis (Jamaica weakfish), Menticirrhus americanus (Southern kingcroaker), Micropogonias furnieri (Whitemouth croaker), Chaetodipterus faber (Atlantic spadefish), Trichiurus lepturus (Largehead hairtail), Balistes capriscus (Grey triggerfish), Stephanolepis hispidus (Planehead filefish) and Chilomycterus spinosus (Southern burrfish).

When considering only the Critical, Endangered and Vulnerable categories, both by the IUCN (2017) criteria and by Brazilian federal Legislation (MMA 2014) we observed a total of 33 threatened species $(13.47 \%)$ in this study. Also, when considering only the threat categories and choosing the most conservative classification, three distinct and worrying scenarios are drawn by correlating the conservation status with the frequency of occurrence (constant, accessory and accidental) (Table 4).
Most of the captured species (35.51\%) during this study were distributed in the Atlantic Ocean (WA), followed by the SSWA (15.10\%), circumtropical (CT) species (13.06\%), Trans-Atlantic (TA) species (11.02\%), Ca+Br (9.8\%), Ca+SWA (4.9\%), SWA (2.45\%), Br (2.04\%), Br+SSWA (2.04\%), and WA+EP (1.63\%). One species $(0.41 \%$ of the total) was found in each of the following areas: TA+EP, SWA+EP, $\mathrm{SSWA}+\mathrm{EP}, \mathrm{Ca}+\mathrm{Br}+\mathrm{EP}, \mathrm{Ca}+\mathrm{SWA}+\mathrm{EP}$, and $\mathrm{Ca}$.

\section{Discussion}

The richness of demersal organisms is directly related to the continental shelf width, sediment types, oceanographic conditions, the geological events that shaped the continental shelf, as well as its positioning relative to the equator characterizing the environmental temperature (Lowe-McConnell 1987, Longhurst \& Pauly 2007).

Although many results of this study can be explained by the oceanographic features of the pair trawling fleet operation area in the state of São Paulo, biotic factors interfere with the species distribution through inter- and intraspecific relations, such as the predator-prey effect and the competitive interactions for food (Sanders 1969, Moyle \& Cech 1998, Menge \& Olson 1990, Barry et al. 1996, Akin et al. 2003).

Overall, the widest and largest continental shelf is in southern Brazil, consisting of muddy sediment and under the influence of the oscillation of the subtropical convergence between the warm waters

Table 4. Checklist of the threatened species captured according to the International Union for Conservation of Nature (IUCN 2017) and the Brazilian list (MMA 2014) and their frequency of occurrence (Dajoz 1983).

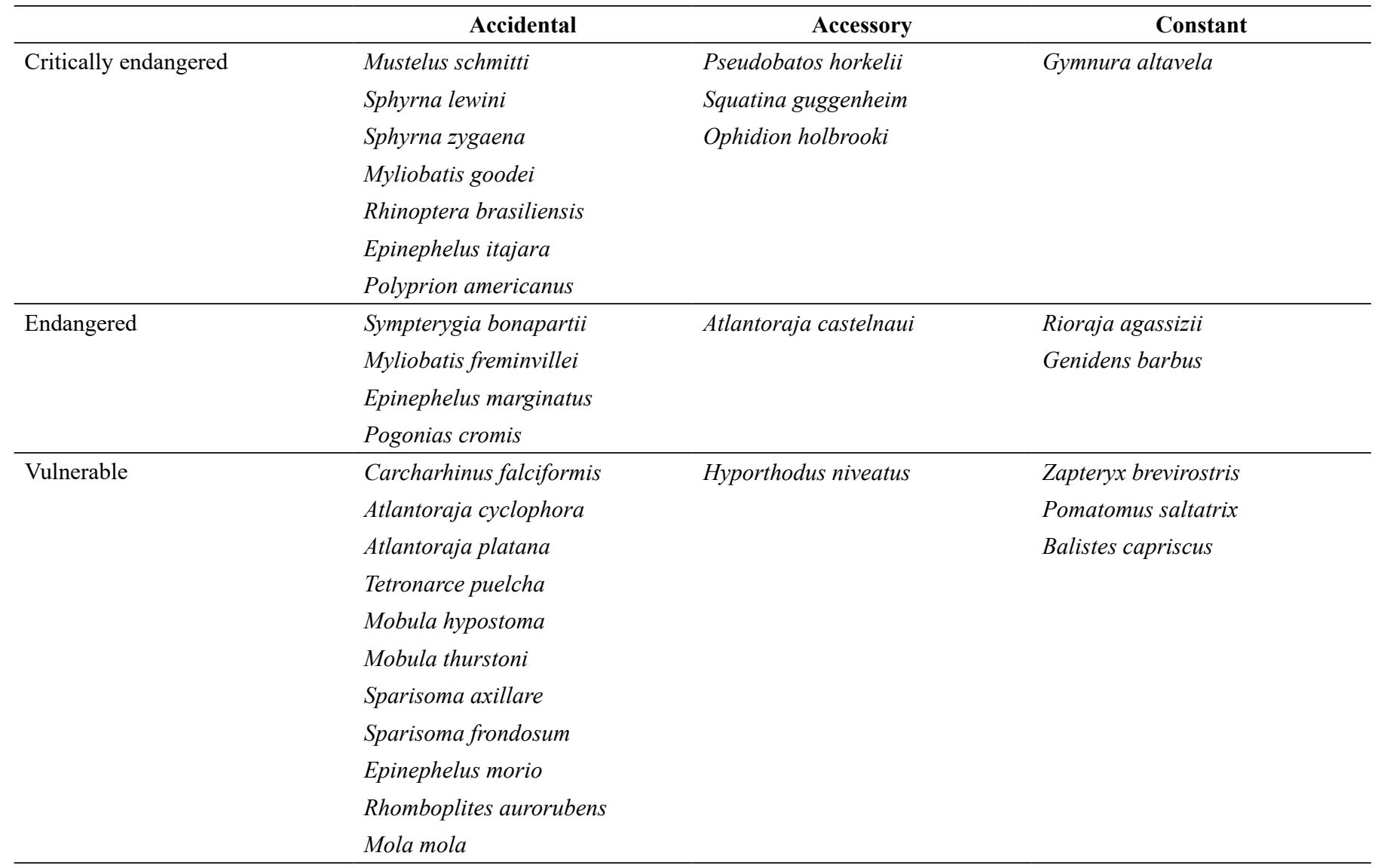


of the Brazilian current and the cold waters of the Malvinas current. The region has upwelling zones that during the summer and spring cause the South Atlantic Central Water (SACW) to penetrate the shelf reaching the coastal areas with a thermocline of 10 and 15 meters. During the winter, SACW retracts and Tropical Water (TW) fills the space. There is significant water input from continental drainage. Due to such characteristics, the region represents the Southern boundary of occurrence of several tropical species (e.g.: Hypanus guttatus, $H$. americanus, Anchoa tricolor, Rypticus randalli) and the Northern boundary of temperate species (e.g.: Atlantoraja castelnaui, A. cyclophora, Sardinella brasiliensis, Boridia grossidens). Moreover, the region has the highest abundance of fishery resources in Brazil (PiresVanin et al. 1993, Matsuura 1995, Castro \& Menezes 1998, Menezes et al. 2003, Braga \& Niencheski 2006, Castro et al. 2006, Castro et al. 2008, Amaral \& Nallin 2011, Menezes 2011).

According to Menezes et al. (2003), 1,297 species of marine fish, belonging to 36 orders and 192 families, occur in Brazil. The results of this study showed the Southern demersal ichthyofauna accounted for $18.9 \%$ of species recorded in Brazil. The families Carangidae, Sciaenidae, Paralichthyidae, Haemulidae, Serranidae and Engraulidae showed high number of species, with most species being demersal, benthic or benthopelagic (Lowe-McConnell 1987).

According to Dajoz's (1983) scale, we could hypothesize that the common richness of the ichthyofauna by landing is approximately 90 species (constant + accessory). However, this value differs from that observed in landings, which ranged from 49 to 104 species (mean and standard deviation $=68.65 \pm 13.20$ ), mainly due to the high number of accidental species.

According to criteria adopted by the International Union for Conservation of Nature (IUCN 2017), species classified as least concern $(64.08 \%)$ were predominant, followed by not-evaluated species $(14.69 \%)$, data deficient $(8.16 \%)$, vulnerable and nearthreatened $(4.90 \%)$, endangered $(2.45 \%)$ and critically endangered $(0.82 \%)$. Based on Brazilian federal legislation (MMA 2014), only $10.61 \%$ of the species are threatened regarding the conservation status: $4.49 \%$ endangered, $3.67 \%$ vulnerable and $2.45 \%$ endangered. Proportionally, Class Chondrichthyes had higher number of highly endangered species $(23.53 \%$ critically endangered, $11.76 \%$ endangered and $20.59 \%$ vulnerable). Based on the three scenarios observed regarding the conservation status of the captured species, the actions of fishery management are priority for the pair trawling fishing operating in the Zoogeographic Province of Argentina. We emphasize this study was carried out in the period before the prohibitions on the capture of several endangered species (MMA 2014); therefore, the continuous monitoring of the composition of this fishing modality is necessary. We also point out there were restrictions of fishing area for pair trawling fishing in the State of São Paulo (SMA 2009). Such restrictions occurred during the last 3 years of this study, which again emphasizes the need for continuous monitoring to verify changes in the composition of the species captured by this type of fishery.

According to Kotas (1991), the pair trawling fishing is characterized as active, multispecies and difficult to manage due to the large number of species captured. The most used management technique in these cases is the restriction of areas and/or periods (High et al. 1969, Caddy 1982), which often causes economic and social problems. There are other ways of ensuring greater selectivity and more sustainability in these fisheries without losing their economic viability, such as adequacy of fishing nets and the use of bycatch reduction devices (BRD). Several BRD models have been developed and tested in different types of environments worldwide, many of which are summarized in the study by Broadhurst (2000). Thus, studies on fishing technology aiming at minimizing the capture of endangered species are essential for biodiversity maintenance.

Caires (2014) listed 120 species (76.67\% Actinopterygii and $23.33 \%$ Chondrichthyes) as endemic to the zoogeographic province of Argentina. Out of the 92 species of Actinopterygii listed, 24 species (26\%) were observed in this study, namely: Conger orbignianus (Congridae), Sardinella brasiliensis (Clupeidae), Merluccius hubbsi (Merlucciidae), Urophycis brasileinsis (Gadidae), Genypterus brasiliensis and Raneya brasiliensis (Ophidiidae), Porichthys porosissimus and Thalassophryne montevidensis (Batrachoididae), Parona signata and Trachinotus marginatus (Carangidae), Sphyraena tome (Sphyraenidae), Etropus longimanus, Paralichthys orbignyanus, P. patagonicus and Xystreurys rasile (Paralichthyidae), Thyrsitops lepidopoides (Gempylidae), Pseudopercis semifasciata (Pinguipedidae), Percophis brasiliensis (Percophidae), Astroscopus sexspinosus (Uranoscopidae), Dules auriga (Serranidae), Boridia grossidens (Haemulidae), Prionotus nudigula (Triglidae), Cynoscion guatucupa and Umbrina canosai (Sciaenidae). Among the 28 species of Chondrichthyes, 11 species $(39.3 \%)$ were captured in the present study, namely: Mustelus schmitti (Triakidae), Squatina guggenheim (Squatinidae), Atlantoraja castelnaui, A. cyclophora, A. platana, Psammobatis extenta, P. lentiginosa, Rioraja agassizii and Sympterygia bonapartii (Rajidae), Pseudobatos horkelii (Rhinobatidae) and Dasyatis hypostigma (Dasyatidae). Due to the distribution (Southeast region of Brazil to Argentina), we also consider the following species endemic to the zoogeographic province of Argentina: Tetronarce puelcha (Torpedinidae), Anchoa marinii and Engraulis anchoita (Engraulidae) and Brevoortia pectinata (Clupeidae). Thus, $35.29 \%$ of Chondrichthyes and $11.85 \%$ of Actinopterygii ( $15.1 \%$ of the total) belong to the endemic fauna of zoogeographic province of Argentina.

The importance of the zoogeographic province of Argentina in the diversity of marine and estuarine fish fauna of Southern Brazil was evidenced in two other studies carried out in the Laje de Santos Marine State Park (Luiz Jr et al. 2008) and in the Paranaguá estuarine complex (Passos et al. 2012). The similarity in species composition was low $(<50 \%)$ due to the different types of environments (rocky reefs, continental shelf and estuary). Totally, 427 species were observed, out of which 46 species $(10.77 \%)$ are endemic to zoogeographic province of Argentina, being the fourth geographic distribution with the highest number of species, only behind the Western Atlantic with 169 species (39.58\%), circumtropical with 52 species $(12.18 \%)$ and trans-Atlantic with 50 species $(11.71 \%)$. There was higher rate of endemism in the zoogeographic province of Argentina among marine demersal species (14.29\%), followed by estuarine (10.95\%) and reef (4.69\%) species.

Based on the results of this work, we highlight the need for studies addressing other aspects of exploited communities, such as diversity analysis, spatial and seasonal distribution, as well as on impacts on explored demersal fishes, such as types of fishing, pollution, degradation of coastal areas and the introduction of exotic species. 


\section{Acknowledgments}

The authors thank all the fishermen from the "Pró-Pesca Project: fishing the knowledge" and the Zoological Collection team at Santa Cecília University.

\section{Author Contributions}

Matheus Marcos Rotundo: collaborated with the identification, data analysis, and writing of the manuscript.

Evandro Severino-Rodrigues: collaborated with the identification, data analysis, and writing of the manuscript.

Walter Barrella: collaborated with the identification, data analysis, and writing of the manuscript.

Miguel Petrere Junior: collaborated with the identification, data analysis, and writing of the manuscript.

Milena Ramires: collaborated with the identification, data analysis, and writing of the manuscript.

\section{Conflicts of interest}

The authors declare that they have no conflict of interest related to the publication of this manuscript.

\section{References}

AKIN, S., WINEMILLER, K.O. \& GELWICK, F.P. 2003. Seasonal and spatial variations in fish and macrocrustacean assemblage structure in Mad Island Marsh estuary, Texas. Estuarine, Coastal and Shelf Science. (57): 269-282.

AMARAL, A.C.Z. \& NALLIN, S.A.H. 2011. Biodiversidade e ecossistemas bentônicos marinhos do Litoral Norte de São Paulo, Sudeste do Brasil. E-Book, UNICAMP, Campinas, 573p. <http://www.bibliotecadigital. unicamp.br/document/?down $=000812694>($ accessed in 15/02/2015).

ARYUTHAKA, C. \& THUBTHIMSANG, W. 1992. Distribution of economically important demersal fish by Depth off Chanthaburi, East Thailand. Thai Mar. Fish. Res. Bull. 3:37-44.

BARRY, J.P., YOKLAVICH, M.M., CAILLIET, G.M., AMBROSE, D.A. \& ANTRIM, B.S. 1996. Trophic ecology of the dominant fishes in Elkhorn Slough, California, 1974-1980. Estuaries. (19): 115-118.

BRAGA, E.S. \& NIENCHESKI, L.F.H. 2006. Composição das massas de água e seus potenciais produtivos na área entre o Cabo de São Tomé (RJ) e o Chuí (RS). In: O ambiente oceanográfico da plataforma continental e do talude na região Sudeste-Sul do Brasil. C.L.D.B. Rossi-Wongtschowski \& L.S.P. Marureira, eds. Edusp. São Paulo, p.161-218.

BROADHURST, M.K. 2000. Modifications to reduce bycatch in prawn trawls: a review and framework for development. Reviews in Fish Biology and Fisheries. 10(1): 27-60.

CADDY, J.F. 1982. Management of shrimp fisheries. In: Fish Bycatch - Bonus from the Sea: Report of a Technical Consultation on Shrimp By-catch Utilization held in Georgetown, Guyana, 27-30 October 1981, IDRC, Ottawa, (IDRC-198e), pp. 120-124.

CADDY, J.F. \& SHARP, G.D. 1986. An ecological framework for marine fishery investigations. FAO Fish. Tech. Pap. (283): 152p.

CAIRES, R.A. 2014. Biogeografia dos peixes marinhos do Atlântico Sul Ocidental: padrões e processos. Arquivos de Zoologia (São Paulo). 45(esp): 5-24

CARPENTER, K.E. 2002. FAO species identification sheets for fishery purposes. The living marine resources of the Western Central Atlantic (fishing area 31) FAO. Roma, V. 1-3.

CARVALHO-FILHO, A. 1999. Peixes da costa brasileira. $3^{\text {a }}$ Ed. Ed. Melro, São Paulo.

CARVALHO-FILHO, A., SANTOS, S., \& SAMPAIO, I. 2010. Macrodon atricauda (Günther, 1880) (Perciformes: Sciaenidae), a valid species from the southwestern Atlantic, with comments on its conservation. Zootaxa. 2519 (4).
CASTEllo, L., CASTEllO, J.P. \& HALL, C.A.S. 2007. Problemas en el estudio y manejo de pesquerías tropicales. Gaceta ecológica. 84-85: 65-73.

CASTRO, B.M., LORENZETTI, J.A., SILVEIRA, I.C.A. \& MIRANDA, L.B. 2006. Estrutura termohalina e circulação na região entre o cabo de São Tomé (RJ) e o Chuí (RS). In: O ambiente oceanográfico da plataforma continental e do talude na região Sudeste-Sul do Brasil. C.L.D.B. Rossi-Wongtschowski \& L.S.P. Marureira, eds. Edusp. São Paulo, p.11 -120.

CASTRO, B.M., MIRANDA, L.B., SILVA, L.S., FONTES, R.F.C., PEREIRA, A.F. \& COELHO, A.L. 2008. Processos Físicos: Hidrografia, Circulação e Transporte. In: Oceanografia de um ecossistema subtropical - Plataforma de São Sebastião, SP (A.M.S. Pires-Vanin, Ed). Edusp. São Paulo, p.59-121.

CASTRO, P.M.G. \& TUTUI, S.L.S. 2007. Frota de parelhas do Estado de São Paulo - caracterização física e operacional, e suas variações temporais. Rev. Bras. Enga. Pesca. 2 (2): 13-29.

CASTRO, P.M.G., CARNEIRO, M.H., SERVO, G.J.M., MUCINHATO, C.M.D. \& SOUZA, M.R. 2003. Dinâmica da pesca de arrasto de parelha do Estado de São Paulo, In: Análise das principais pescarias comerciais do sudeste-sul do Brasil: Dinâmica das frotas pesqueiras. (M.C. Cergole \& C.L.D.B. RossiWongtschowski, eds). REVIZEE/MMA/SECIRM/FEMAR. Brasília, p.65-115.

CASTRO, P.M.G.; CARNEIRO, M.H.; SERVO, G.J.M.; MUCINHATO, C.M.D.; SOUZA, M.R. 2007. Dinâmica da frota de arrasto de parelhas do Estado de São Paulo, In: Dinâmica das Frotas Pesqueiras Comerciais da Região Sudeste - Sul do Brasil (C.L.D.B. Rossi-Wongtschowski, R.A. Bernardes \& M.C. Cergole, eds). REVIZEE/MMA/SECIRM/FEMAR. Brasília, p.60-103.

CASTRO, R.M.C. \& MENEZES, N.A. 1998. Estudo Diagnóstico de Diversidade de Peixes do Estado de São Paulo. In: Biodiversidade do Estado de São Paulo, Brasil: síntese do conhecimento ao final do século XX-6: Vertebrados. (C.A. Joly \& C.E.M. Bicudo, eds). Fapesp. São Paulo, p.1-13.

CERVIGÓN, F., CIPRIANI, R., FISCHER, W., GARIBALDI, L., HENDRICKX, M., LEMUS, A.J., MÁRQUEZ, R., POUTIERS, J.M., ROBAINA, G. \& RODRIGUEZ, B. 1992. Guia de campo de las especies comerciales marinas y de aguas salobres de la costa septentrional de Sur America. Organización de las Naciones Unidas para la Agricultura y la Alimentación, Roma.

COMPAGNO, L.J.V. 1984. FAO Species catalogue Vol 4. Sharks of the world. An annotated and illustrated catalogue of shark species known to date. Parts 1, 2/ FAO Fish. Synopsis. (125) vol 4, Roma.

COMPAGNO, L. J.V. 1988. Sharks of the Order Carcharhiniformes. Princeton University Press, New Jersey.

DAJOZ, R. 1983. Ecologia Geral. EDUSP, São Paulo.

DAVIS, G.E. \& ANDERSON, T.W. 1989. Populations estimatives of four kelp forest fishes and an evaluation of three in situ assessment techniques. Bull. Mar. Sci. 44 (3): 1138-1151.

DIAS-NETO, J. \& XIMENES, M.J. 1998. Potencialidades e exploração dos recursos pesqueiros do Brasil. Ciência e Cultura. 40 (5): 427-441.

ESCHMEYER, W.N., FRICKE, R. \& VAN DER LAAN, R. 2017. Catalog of fishes: genera, species, references. (http://researcharchive.calacademy.org/ research/ichthyology/catalog/fishcatmain.asp). (last access on 10/08/2017).

FIGUEIREDO, J.L. 1977. Manual de peixes marinhos do sudeste do Brasil. Introdução, cações, raias e quimeras. Museu de Zoologia da Universidade de São Paulo, São Paulo.

FIGUEIREDO, J.L. \& MENEZES, N.A. 1978. Manual de Peixes marinhos do sudeste do Brasil.2 Teleostei (1). Museu de Zoologia da Universidade de São Paulo, São Paulo.

FIGUEIREDO, J.L. \& MENEZES, N.A. 1980. Manual de peixes marinhos do sudeste do Brasil.3 Teleostei (2). Museu de Zoologia da Universidade de São Paulo, São Paulo.

FIGUEIREDO, J.L. \& MENEZES, N.A. 2000. Manual de peixes marinhos do sudeste do Brasil.6 Teleostei (5). Museu de Zoologia da Universidade de São Paulo, São Paulo.

FISCHER, L.G., PEREIRA, L.E.D. \& VIEIRA, J.P. 2004. Peixes estuarinos e costeiros: Série Biodiversidade do Atlântico Sudoeste 01. Editora Coscientia, Rio Grande.

FISCHER, W. 1978. FAO species identification sheets for fishery purposes. Western Central Atlantic (fishing area 31). FAO, Roma. 
FLOETER, S.R., ROCHA, L.A., ROBERTSON, D.R., JOYEUX, J.C., SMITH-VANIZ, W.F., WIRTZ, P., EDWARDS, A.J., BARREIROS, J.P., FERREIRA, C.E.L., GASPARINI, J.L., BRITO, A., FALCÓN, J.M., BOWEN, B.W. \& BERNARDI, G. 2008. Atlantic reef fish biogeography and evolution. J. Biogeogr. 35:22-47.

FRABLE, B.W., BALDWIN, C.C., LUTHER, B.M. \& WEIGT, L.A. 2013. A new species of western Atlantic lizardfish (Teleostei: Synodontidae: Synodus) and resurrection of Synodus bondi Fowler, 1939, as a valid species from the Caribbean with redescriptions of $S$. bondi, S. foetens (Linnaeus, 1766), and S. intermedius (Agassiz, 1829). Fishery Bulletin. 111 (2): 122-146.

GOMES, U.L., SIGNORI, C.N., GADIG, O.B.F. \& SANTOS, H.R.S. 2010. Guia para a identificação de tubarões e raias do Rio de Janeiro. Technical Books, Rio de Janeiro.

HAIMOVICI, M. 1997. Recursos Pesqueiros Demersais da Região Sul. FEMAR, Rio de Janeiro.

HIGH, W.L., ELLIS, I.E. \& LUSZ, L.D. 1969. A progress report on the development of a shrimp trawl to separate shrimp from fish and bottomdwelling animals. Commer. Fish. Rev. 31, 20-33.

IBAMA. 1993. Peixes Demersais: Relatório da III Reunião do Grupo Permanente de Estudos sobre Peixes Demersais. IBAMA, Brasília.

IBAMA. 1995. Peixes Demersais: Relatório da $4^{a}$ Reunião do Grupo Permanente de Estudos, 08 - 12 nov, 1993. IBAMA/ CEPSUL, Itajaí.

IUCN. 2017. The IUCN red list of threatened species. International ed. IUCN Global Species Programme Red List Unit. Available: http://www.iucnredlist. org, (accessed in 5/12/2017).

KNUDSEN, S.W. \& CLEMENTS, K.D. 2016. World-wide species distributions in the family Kyphosidae (Teleostei: Perciformes). Molecular phylogenetics and evolution. 101, 252-266.

KOTAS, J.E. 1991. Análise dos desembarques da pesca industrial de arrasteiros de parelha sediados nos municípios de Itajaí e Navegantes (SC) durante o ano de 1986. Atlântica, Rio Grande, 13(1): 97-105.

LAST, P.R., NAYLOR, G.J. \& MANJAJI-MATSUMOTO, B.M. 2016. A revised classification of the family Dasyatidae (Chondrichthyes: Myliobatiformes) based on new morphological and molecular insights. Zootaxa. 4139 (3): 345-368.

LAST, P.R., SERET, B. \& NAYLOR, G.J. 2016. A new species of guitarfish, Rhinobatos borneensis sp. nov. with a redefinition of the familylevel classification in the order Rhinopristiformes (Chondrichthyes: Batoidea). Zootaxa. 4117(4): 451-475.

LAST, P.R., WHITE, W., SÉRET, B., NAYLOR, G., DE CARVALHO, M. \& STEHMANN, M. 2016. Rays of the World. CSIRO Publishing, New York.

LEIS, J.M. 2006. Nomenclature and distribution of the species of the porcupinefish family Diodontidae (Pisces, Teleostei). Memoirs of Museum Victoria. 63 (1): 77-90.

LONGHURST, A.R. \& PAULY, D. 2007. Ecologia de Oceanos Tropicais. Edusp, São Paulo.

LOWE-McCONNEL, R.H. 1987. Ecological studies in tropical fish communities. Cambridge University Press, Cambridge.

LUIZ Jr, O.J., CARVALHO-FILHO, A., FERREIRA, C.E.L., FLOETER, S.R., GASPARINI, J.L. \& SAZIMA, I. 2008. The reef fish assemblage of the Laje de Santos Marine State Park, Southwestern Atlantic:annotated checklist with comments on abundance, distribution, trophic structure, symbiotic associations, and conservation. Zootaxa. 1807:1-25.

MCBRIDE, R.S., ROCHA, C.R., RUIZ-CARUS, R. \& BOWEN, B.W. 2010. A new species of ladyfish, of the genus Elops (Elopiformes: Elopidae), from the western Atlantic Ocean. Zootaxa. 2346: 29-41.

MARCENIUK, A.P. 2005. Chave para a identificação das espécies de bagres marinhos (Siluriformes, Ariidae) da Costa Brasileira. Boletim do Instituto de pesca. 31 (2): 89-101.

MARCENIUK, A. P., CAIRES, R., SICCHA-RAMIREZ, R. \& OLIVEIRA, C. 2016. Review of the harvestfishes, genus Peprilus (Perciformes: Stromateidae), of the Atlantic coast of South America. Zootaxa. 4098 (2): 311-332.
MATSUURA, Y. 1995. Exploração pesqueira - ambiente marinho no litoral brasileiro In: Os ecossistemas brasileiros e os principais macrovetores de desenvolvimento: subsídios ao planejamento da gestão ambiental (Secretaria de Coordenação dos Assuntos de Meio Ambiente). MMA, Brasília. p.1-15.

MENEZES, N.A. 2011. Checklist of marine fishes from São Paulo State, Brazil. Biota Neotrop. 11(1). Available: <http://www.biotaneotropica.org. br/v11n1a/pt/fullpaper?bn0031101a2011+pt> (accessed in: 15/01/2012).

MENEZES, N.A., BUCKUP, P.A., FIGUEIREDO, J.L. \& MOURA, R.L. 2003. Catálogo das espécies de peixes marinhos do Brasil. Museu de Zoologia da Universidade de São Paulo, São Paulo.

MENEZES, N.A. \& FIGUEIREDO, J.L. 1980. Manual de peixes marinhos do sudeste do Brasil.IV.Teleostei (3). Museu de Zoologia da Universidade de São Paulo, São Paulo.

MENEZES, N.A. \& FIGUEIREDO, J.L. 1985. Manual de peixes marinhos do sudeste do Brasil.V. Teleostei (4). Museu de Zoologia da Universidade de São Paulo, São Paulo.

MENEZES, N.A., NIRCHIO, M., DE OLIVEIRA, C. \& SICCHARAMIREZ, R. 2015. Taxonomic review of the species of Mugil (Teleostei: Perciformes: Mugilidae) from the Atlantic South Caribbean and South America, with integration of morphological, cytogenetic and molecular data. Zootaxa. 3941 (4): 1-38.

MENGE, B.A. \& OLSON, A.M. 1990. Role of scale and environmental factors in regulation of community structure. Trends in Ecology and Evolution. 5: 52-57.

MMA. 2014. Portaria $\mathrm{n}^{\circ} 445$, de 17 de dezembro de 2014. (accessed in $15 / 12 / 2017)$.

MOURA, R.L., FIGUEIREDO, J.L. \& SAZIMA, I. 2001. A new parrotfish (Scaridae) from Brazil, and revalidation of Sparisoma amplum (Ranzani, 1842), Sparisoma frondosum (Agassiz, 1831), Sparisoma axillare (Steindachner, 1878) and Scarus trispinosus Valenciennes, 1840. Bulletin of Marine Science. 68(3): 505-524.

MOURA, R.L. \& LINDEMAN, K.C. 2007. A new species of snapper (Perciformes: Lutjanidae) from Brazil, with comments on the distribution of Lutjanus griseus and L. apodus. Zootaxa. 1422 (3): 31-43.

MOYLE, P.B. \& CECH, J.J.1998. Fishes: An introduction to ichthyology. 2ed. Prentice Hall. Englewood Cliffs, NJ, USA.

NELSON, J.S., GRANDE, T.C. \& WILSON, M.V. 2016. Fishes of the World. John Wiley \& Sons. Hoboken, New Jersey, USA.

PASSOS, A.C.D., CONTENTE, R.F., ARAUJO, C.C.V.D., DAROS, F.A.L.D.M., SPACH, H.L., ABILHÔA, V. \& FÁVARO, L.F. 2012. Fishes of Paranaguá estuarine complex, south west Atlantic. Biota Neotropica, 12(3): 226-238.

PIRES-VANIN, A.M.S., ROSSI-WONGSTSHOWSKI, C.L.D.B., AIDAR, E.; MESQUITA, H.S.L., SOARES, L.S.H., KATSURAGAWA, M. \& MATSUURA, Y. 1993. Estrutura e função do ecossistema de plataforma continental do Atlântico Sul brasileiro: síntese dos resultados. Publ. Esp. Inst. Oceanogr. 10: 217-231.

ROSA, M.R. \& GADIG, O.B.F. 2010. Taxonomic comments and an identification key to species for the Smooth-hound sharks genus Mustelus Link, 1790 (Chondrichthyes: Triakidae) from the Western South Atlantic. Pan-American Journal of Aquatic Sciences. 5 (3): 401-413.

RUOCCO, N.L., LUCIFORA, L.O., DE ASTARLOA, J.D., MABRAGAÑA, E. \& DELPIANI, S.M. 2012. Morphology and DNA barcoding reveal a new species of eagle ray from the southwestern Atlantic: Myliobatis ridens sp. nov. (Chondrichthyes: Myliobatiformes: Myliobatidae). Zoological Studies. 51 (6): 862-873.

SAMPAIO, C.L.S. \& NOTTINGHAM, M.C. 2008. Guia para identificação de peixes ornamentais brasileiros. Volume I: espécies marinhas. IBAMAMMA, Brasília.

SANDERS, H.L. 1969. Marine benthic diversity: a comparative study. American Naturalist. 102: 243-282.

SMA. 2009. Resolução n.69 de 28 de setembro de 2009. (accessed in 15/12/2017).

TAVERA, J.J., PIZARRO, A.A., LA CRUZ-AGÜERO, D. \& BALART, E.F. 2011. Phylogeny and reclassification of the species of two neotropical grunt genera, Anisotremus and Genyatremus (Perciformes: Haemulidae), based on morphological evidence. Journal of Zoological Systematics and Evolutionary Research. 49 (4): 315-323. 
Rotundo, M.M. et al.

TAVERA, J.J., ACERO, A., BALART, E.F. \& BERNARDI, G. 2012. Molecular phylogeny of grunts (Teleostei, Haemulidae), with an emphasis on the ecology, evolution, and speciation history of New World species. BMC evolutionary biology. 12(1): 57.

VALENTINI, H., CASTRO, P.M. G., SERVO, G.J.M. \& CASTRO, L.A.B. 1991. Evolução da pesca das principais espécies demersais da costa sudeste do Brasil, pela frota de arrasteiros de parelha baseada em São Paulo, de 1968 a 1987. Atlântica. 13 (1): 87-95.

YÁÑES-ARANCIBIA, A. \& SÁNCHEZ-GIL, P. 1988. Ecologia de los recursos demersales marinos. Ed. México, D.F.
YAÑEZ-ARANCIBIA, A., SANCHEZ-GIL, P., TAPIA-GARCIA, M. \& GARCIA-ABAD, M.C.1985. Ecology, community structure and evaluation of tropical demersal fishes in southern Gulf of Mexico. Cahiers de Biologie Marine. 26 (2): 137-163.

Received: $2208 / 2018$

Revised: $23 / 11 / 2018$

Accepted: 12/12/2018

Published online: 21/01/2019 